\title{
stockages souterrains, enfouissement et environnement
}

\author{
Rapport général de \\ $\mathrm{Ph}$. Masure \\ Chef du Département de Génie Géologique \\ Bureau de Recherches Géologiques et Minières - Orléans
}

\section{Introduction}

Les premières traces de l'exploitation des ressources du sous-sol par I'homme remontent à quelques 5000 ans. Des puits d'exploitation de silex de 10 à $15 \mathrm{~m}$ de profondeur reliés entre eux par des galeries ont été excavés à cette époque reculée en Belgique et en Hollande près de Maastricht sur de nombreux hectares. Depuis, l'exploitation des ressources minérales, de l'eau souterraine, des métaux et des substances organiques solides, liquides et gazeuses s'est grandement développée, accompagnant l'expansion des sociétés agricoles puis industrielles. Aujourd'hui, les ressources géothermiques, dont les manifestations nombreuses étaient connues depuis l'Antiquité, commencent à faire l'objet d'une exploitation planifiée dans notre pays.

De leur côté, les immenses ressources en espace souterrain n'ont retenu l'attention des sociétés que de manière diffuse durant des millénaires et ont été développées, comme les autres ressources du soussol, par nécessité. C'est la maîtrise de l'eau qui a d'abord exigé des tunnels à travers les montagnes [Duffaut, 1981]. Le premier fût creusé à Samos il y a 2600 ans par Eupalinos pour alimenter la ville avec une source lointaine en cas de siège. Les tunnels de communication suivirent beaucoup plus tard.

Le stockage souterrain de matières consommables, donc récupérables, a suivi la même évolution. Constituant d'abord une facilité pour I'homme préhistorique, redécouverte par l'homme moderne, cette activité devient aujourd hui une nécessité de plus en plus impérative. Nécessité de préserver l'espace superficiel, nécessité de limiter la pollution et les nuisances, nécessité d'accroître la sécurité des installations. Les problèmes posés par l'évolution du milieu récepteur et son environnement au cours du stockage ont donné lieu à des études scientifiques et techniques spécifiques (mécaniques, thermiques et hydrauliques) qui ont permis de maîtriser avec une fiabilité suffisante l'impact de tels projets sur l'environnement.

La situation est bien différente en matière d'évacuation de déchets de grande nocivité dans le sous-sol. La production de certains déchets industriels toxiques a atteint un niveau tel que leur dispersion dans le sol et les eaux n'est plus biologiquement satisfaisante. Leur transformation ou leur isolement définitif de la biosphère s'impose. Les projets d'évacuation définitive de substances toxiques par enfouissement dans le sous-sol constituent aujourd'hui un pari scientifique audacieux. Si la réussite de cette dernière entreprise n'est pas encore totalement assurée, elle a déjà permis de faire d'utiles réflexions sur la recherche et la sélection de sites de confinement et sur la nécessité d'une gestion rationnelle de l'espace souterrain.

Le géotechnicien joue un rôle actif à tous les niveaux de l'aménagement : dans les études de planification ou d'occupation de l'espace qui doivent tenir compte des contraintes comme des potentialités du milieu naturel: dans les études spécifiques d'infrastructure et d'équipement, de la conception à la réalisation des projets; dans les études d'impact qui visent à préserver - voire améliorer - la qualité du milieu; dans la surveillance et le contrôle des ouvrages durant leur exploitation et après leur fermeture, afin d'assurer la sécurité des personnes et des biens. Son domaine d'action se limite toutefois au milieu géologique - à la géosphère - aux côtés de nombreuses autres spécialités des sciences et de la technique. De ce fait, son rôle ne sera jamais aussi complet et prédominant que dans le domaine de la conquête de l'espace souterrain, dont il doit favoriser l'occupation sûre et rationnelle par une bonne connaissance de l'environnement géologique et de son comportement. Trop souvent limité à un rôle très spécifique et ponctuel dans des projets de génie civil, il a une vocation de maître d'œuvre en matière d'aménagement et de gestion de l'espace souterrain. 


\section{Le stockage souterrain des matières con-} sommables

En 1980 , notre pays a consommé 108 millions de tonnes de pétrole et 24 milliards de mètres cubes de gaz naturel. Pour des raisons économiques et stratégiques évidentes, la France est actuellement contrainte de stocker 30 millions de mètres cubes de pétrole et 4 milliards de mètres cubes normaux de gaz naturel. Ce stock de gaz devra être doublé en 1985 , triplé en 1990. Le recours classique à de grandes cuves de 50 mètres de diamètre et 20 mètres de hauteur nécessiterait un équipement d'une centaine de milliers de gazomètres. Leur emprise au sol serait de plusieurs centaines de kilomètres carrés. Le stockage souterrain ne constitue plus alors une simple alternative, comme il l'a longtemps été en matière d'aménagement, mais devient une nécessité impérieuse.

Onze réservoirs souterrains de gaz naturel existent déjà dans notre pays, dont le premier - celui de Beynes, dans les Yvelines - a été mis en charge dès 1956 (fig. 1). La recherche de sites favorables se poursuit activement dans les principaux bassins sédimentaires: bassins parisien, aquitain, méditerranéen, Alsace-Lorraine. En moyenne, quatre sites potentiellement favorables doivent être reconnus pour un site définitivement retenu par Gaz de France.

Cet effort important réalisé par les pays industrialisés ne se développe pas uniquement pour le stockage du gaz naturel, mais également pour de nombreux autres produits :

- pour le pétrole et ses dérivés liquides (naphta, fuel, essence, etc.),

- pour les produits chimiques de base (éthylène, propylène, ammoniac, etc.) ou les gaz de pétrole liquéfiès (butane, propane)

- pour l'eau chaude d'origine industrielle,

- pour l'air comprimé,

- pour les produits solides divers, y compris militaires.

Les stockages souterrains présentent en fait de nombreux avantages. En premier lieu, ils épargnent l'espace superficiel: un stockage souterrain d'un demi-milliard de mètres cubes de gaz présente une emprise au sol de 30 hectares seulement. La dégradation du paysage en est très limitée. La sécurité, vis-à-vis d'agressions extérieures ou d'accidents (chute d'avions par exemple), est très largement améliorée. II en est de même pour la sécurité de l'installation au cours de l'exploitation, notamment pour le stockage de gaz liquéfiés sous pression. D'une manière générale, la protection de l'environnement écologique et de l'homme est respectée avec une qualité qu'il est rare de rencontrer dans les activités industrielles. En outre, lorsque l'alternative est possible, le stockage souterrain s'avère plus économique que le stockage superficiel, à partir d'une certaine dimension.

Ces aspects positifs ne doivent pas occulter cependant un certain nombre de difficultés que l'on connaît encore aujourd'hui, faute d'expérience et de maîtrise suffisante des connaissances sur la structure et le comportemient du sous-sol. On ne domine pas encore dans tous ses détails la faisabilité technique des installations de stockage souterrain, ce qui se traduit par des incertitudes sur les délais d'exécution et les coûts, par des difficultés dans les conditions de

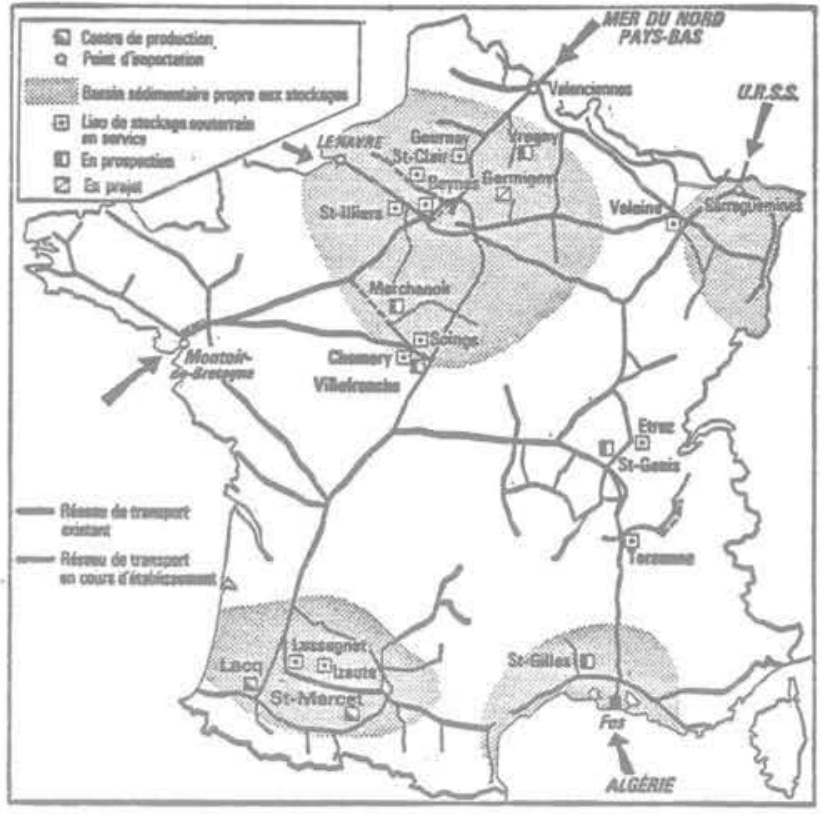

Fig. 1

passation des marchés. On doit correctement apprécier et prévenir les impacts possibles sur la surface du sol, sur l'eau souterraine, sur les massifs récepteurs eux-mêmes, ce qui nécessite des reconnaissances et études nombreuses. On doit enfin adapter les règlements administratifs à ce type nouveau d'occupation de l'espace.

Toutefois, il est clair que l'expérience acquise au cours de ces dernières décennies, notamment en matière de stockage d'hydrocarbures, a permis de dominer le problème de manière satisfaisante et d'assurer un respect rigoureux de l'environnement géologique, une préservation remarquable de l'environnement écologique.

Le stockage de gaz naturel (tabl. I) se fait généralement au sein d'aquifères dans lesquels toutes les conditions d'un gisement naturel sont rencontrées (fig. 2) : un niveau récepteur suffisamment poreux et perméable, surmonté d'un niveau imperméable à concavité dirigée vers le bas (structure anticlinale), assurant ainsi le piégeage du gaz. Des volumes énormes peuvent ainsi être stockés.

Le stockage peut également se faire en cavités salines qui sont creusées par simple lessivage du sel à l'eau douce (fig. 3). Cette technique, très répandue dans le monde (2000 cavités dans le sel, pour une soixantaine en France : Lacoste et Berrest, 1981) est utilisée pour tous les hydrocarbures liquides, gazeux ou liquéfiés. Les cavités lessivées peuvent avoir jusqu'à 200 ou 300 mètres de hauteur (Manosque) et des diamètres supérieurs à 50 mètres.

Le stockage en cavités minées fermées, enfin, peut se satisfaire dans des cas favorables, d'anciennes exploitations minières : c'est le cas célèbre de May-sur-Orne (Géostock). On évalue aujourd'hui la possibilité d'utiliser des karsts pour du gaz. Mais, dans la plupart des cas, on est contraint de créer spécialement des cavités de grand volume. Ce type de stockage a été remarquablement développé en Suède grâce à la qualité du sous-sol rocheux du bouclier scandinave. De par son coût de réalisation élevé, cette technique n'est attractive en France que pour les produits liquides ou liquéfiables. On doit noter cependant un intérêt nouveau porté aux mines et carrières abandonnées pour le stockage de produits solides. 
Tableau I

Volumes des stockages souterrains français (1979)

(d'après Lacoste et Bérest)

\begin{tabular}{|c|c|c|c|c|c|c|c|}
\hline Type de stockage & Gaz naturel & $10 \mathrm{kWh}$ & $10^{6} \mathrm{NM}^{3}$ & Pétrole & $10^{3} \mathrm{~m}^{3}$ & Gaz liquéfie & $10^{3} \mathrm{~m}^{3}$ \\
\hline Aquifères & $\begin{array}{l}\text { Beynes } \\
\text { Saint-Illiers } \\
\text { Chemery } \\
\text { Velaine } \\
\text { Gournay } \\
\text { Lussagnet }\end{array}$ & $\begin{array}{r}4,11 \\
6,4 \\
15,7 \\
5,3 \\
4,2 \\
4,4\end{array}$ & $\begin{array}{r}370 \\
580 \\
1400 \\
480 \\
380 \\
400\end{array}$ & & & & \\
\hline $\begin{array}{l}\text { Cavités salines } \\
\text { lessivées }\end{array}$ & Tersannes & 1,7 & 150 & Manosque & 7000 & $\begin{array}{l}\text { Viriat } \\
\text { Grand Serre } \\
\text { Carresse }\end{array}$ & $\begin{array}{r}120 \\
70 \\
44\end{array}$ \\
\hline Cavités minées & & & & May-sur-Orne & 5000 & $\begin{array}{l}\text { Donges } \\
\text { Lavéra } \\
\text { Porcheville } \\
\text { Petit-Couronne }\end{array}$ & $\begin{array}{r}80 \\
125 \\
130 \\
65\end{array}$ \\
\hline
\end{tabular}

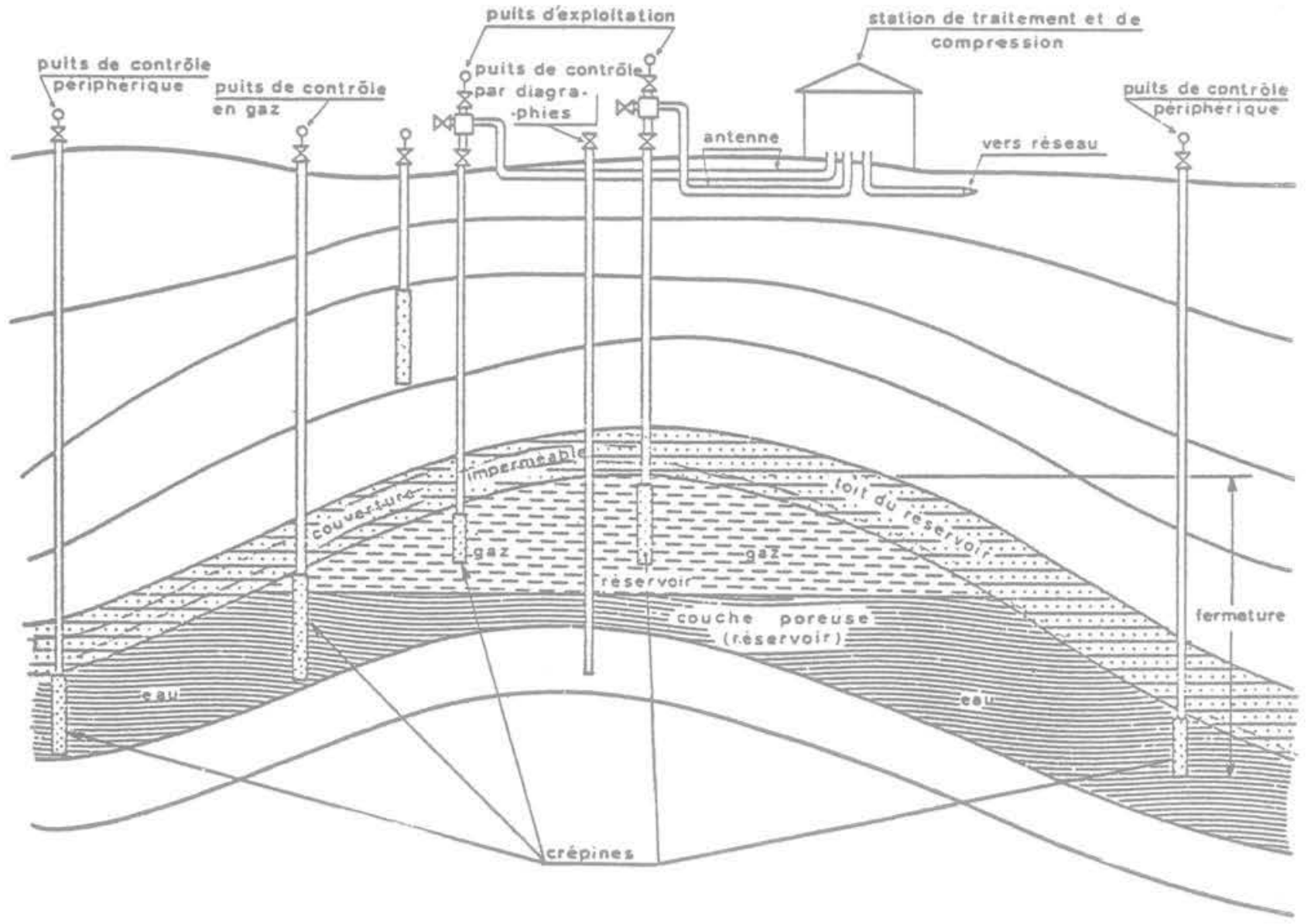

Fig. 2 Coupe schématique d'un réservoir souterrain en nappe aquifère

Les principales incertitudes auxquelles se heurtent les études de faisabilité de projets de stockages souterrains ne sont pas nouvelles pour les géotechniciens. Elles sont de trois ordres:

(i) imprécision dans la définition de la structure interne des massifs rocheux, dans la détermination de leurs caractéristiques physiques, hydrauliques et mécaniques et dans la simulation de leurs équilibres internes,

(ii) difficulté d'appréciation des effets d'échelle d'espace et de temps (comportement à long terme, fluage en masse). (iii) difficulté de caractériser de manière précise et anticipée l'impact du stockage sur la géosphère.

Face à ces incertitudes inhérentes aux opérations nouvelles en matière de génie géologique, les programmes de conception et de réalisation de stockages souterrains se sont déroulés de manière pragmatique en mêlant, après une reconnaissance détaillée des sites sélectionnés, approche théorique et observations pratiques à grande échelle. La méthode appliquée consiste en une sélection et une adaptation 

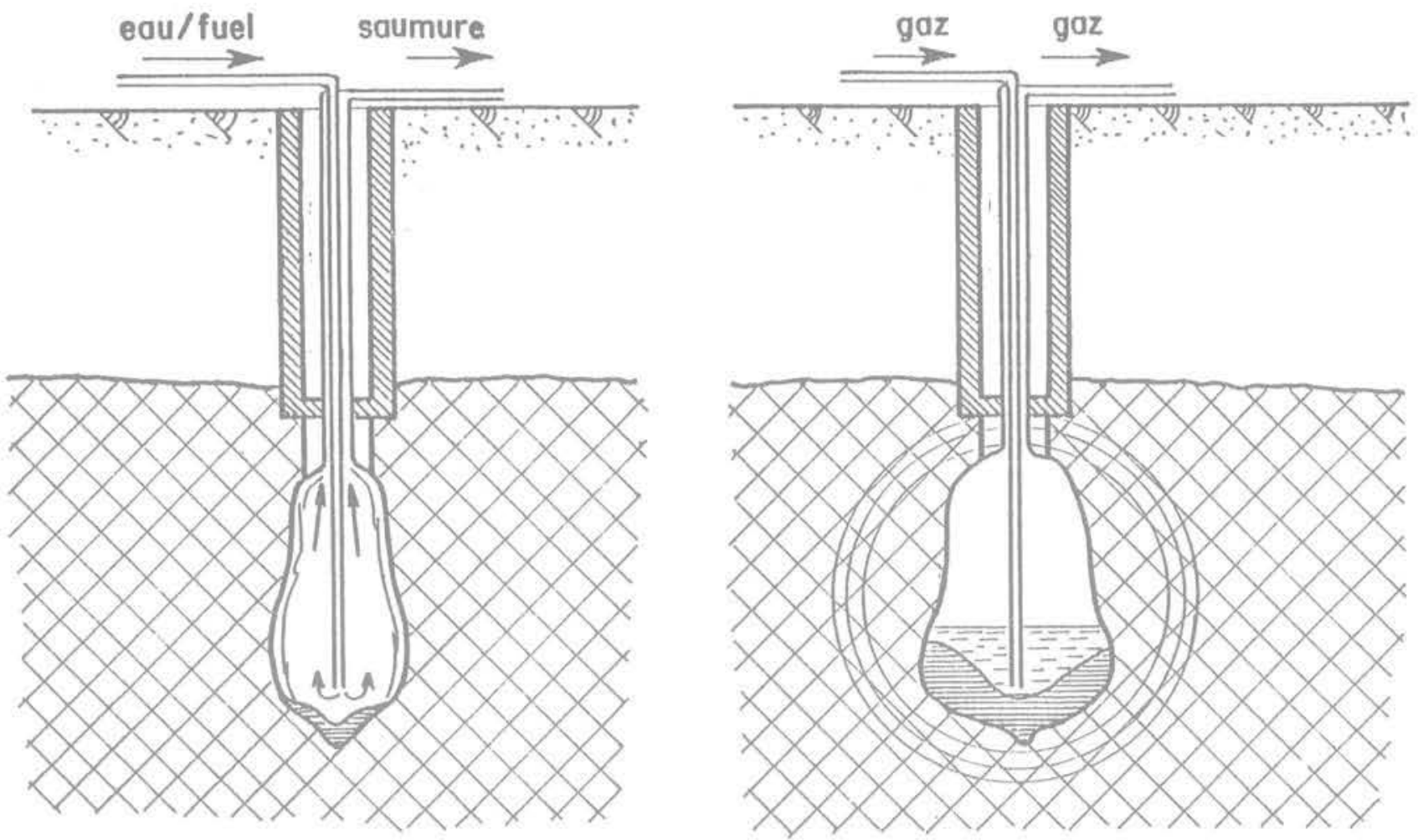

Fig. 3

progressive du projet aux conditions réelles du site, avec traitement éventuel du milieu naturel, pour en corriger les "défauts".

Dans cette démarche, l'évaluation des contraintes (géostatiques, hydrauliques, thermiques, dynamiques) et des écoulements (exhaure, risques de dénoyage, confinement des produits liquides ou gazeux) et leur observation et mesure "in situ " revêtent une importance fondamentale pour les stockages en cavités artificielles. Tout comme le maintien des formes et des tracés fixés, ainsi que le respect de l'intégrité du massif au cours de la réalisation des ouvrages, dans un but fonctionnel. Tout comme l'auscultation détaillée de l'ensemble massif-ouvrage au cours de l'exploitation, dans un but de sécurité.

De la même manière, les stockages en aquifères respectent diverses étapes techniques dont chacune est ponctuée par une analyse détaillée : par exemple, forage de reconnaissance, essais de pompage, injection expérimentale, essais d'étanchéité, mise en service avec forages de contrôle.

II est clair que l'expérience accumulée aujourd'hui a fortement contribué à la maîtrise de la stabilité des cavités et de l'étanchéité des stockages, durant toute la période d'exploitation. Lacoste et Bérest (1981) apportent des précisions intéressantes sur le comportement observé des cavités salines. Des pertes de volume importantes ont pu être notées lorsque le gradient des pressions entre le stockage et le terrain est élevé (supérieur à $20 \mathrm{MPa}$ environ). Ceci confirme le comportement visqueux du sel gemme, mais révèle des capacités de fluage nettement plus élevées que celles que l'on peut généralement définir au laboratoire. En particulier, le sel gemme ne peut supporter durablement un écart important à l'état de contrainte isotrope. La cavité de Tersanne (1400 mètres de profondeur) a ainsi perdu $30 \%$ de son volume après dix ans d'exploitation. L'expérience française incite aujourd'hui à ne pas implanter les cavités salines trop profondément, l'optimum se situant entre 800 et 1200 mètres. En évitant des toits plats de grande portée, la bonne tenue de la partie supérieure des cavités est assurée. Le développement de plusieurs cavités de stockage dans un même massif salin est possible si un écartement entre cavités égal à trois ou quatre fois leur diamètre maximum est respecté.

Les mêmes auteurs, tout comme V. Maury (1981), précisent également les conditions qui assurent l'étanchéité des stockages en cavités rocheuses non revêtues, ou plutôt, le confinement des produits liquides ou gazeux stockés. La première de ces conditions consiste bien évidemment à maintenir une dépression sensible entre la pression de l'eau dans le massif et la pression interne des produits stockés. Alors que l'eau qui pénètre en permanence dans la cavité doit être pompée, il convient quelquefois de la réinjecter dans le terrain afin que n'apparaisse une dépression dangereuse à la surface libre de l'aquifère. voire un dénoyage du massif. La deuxième condition a trait à ce que les auteurs appellent "l'appel d'eau". L'arrivée d'eau doit être effective sur l'ensemble du pourtour de la galerie. Ce phénomène peut être rendu très complexe par la hauteur des cavitès, le cheminement préférentiel de l'eau le long de certaines discontinuités, la poussée d'Archimède sur le produit stocké et, en sens inverse, par les effets capillaires des parois. Pour cela, une dépression minimale tenant compte des caractéristiques physiques des cavités doit être respectée. Seule l'administration française semble s'être préoccupée de ce problème qui est à présent bien contrôlé. La socièté Géostock dispose à ce sujet 


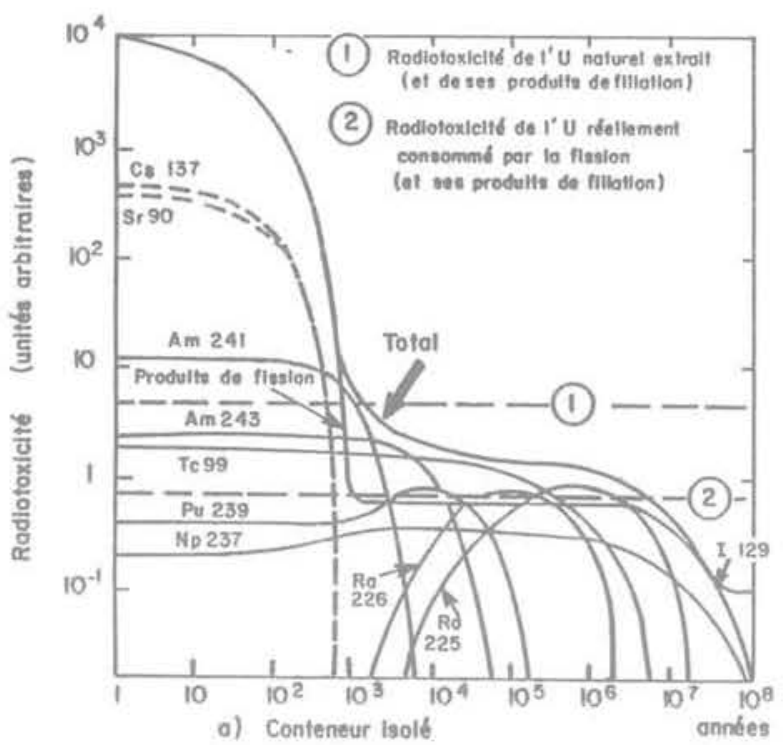

Fig. 4

d'une méthodologie pratique appelée "détermination du coefficient de formen. A titre d'exemple, la dépression de sécurité pour un stockage de propane en cavité de hauteur modérée est de l'ordre de 30 mètres d'eau.

Si l'expérience acquise permet de maîtriser la sécurité de la plupart des projets de stockage souterrain, les géotechniciens n'ont pas encore su vaincre certaines difficultès présentées par les stockages cryogéniques. Le stockage souterrain de propylène liquéfié, à une température de $-40^{\circ} \mathrm{C}$, est aujourd'hui développé industriellement. Par contre, les tentatives de stockage d'éthylène à $-101^{\circ} \mathrm{C}$ ou de gaz naturel à $-162^{\circ} \mathrm{C}$ ont jusqu'ici échoué, bien que des études soient menées activement depuis plusieurs années dans divers pays, notamment aux États-Unis, en Suède et au Japon dans du granite, en Allemagne fédérale dans du sel. Le dépassement de la résistance à la traction à la paroi refroidie du stockage, sous l'effet du gradient thermique, favorise le développement de fissures, généralement perpendiculaires à la contrainte principale mineure. Le liquide pénètre alors dans les fissures, se vaporise et les propage indéfiniment, avec l'inconvénient supplémentaire d'augmenter les pertes énergétiques du système. Un important champ de recherche appliquée est encore largement ouvert dans ce domaine.

Les stockages souterrains de calories, auparavant négligées (rejets industrieis) ou trop coûteuses à produire (solaire), suscitent depuis quelques années un intérêt croissant, notamment de par la souplesse d'utilisation intersaisonnière qu'ils permettent. IIs demandent toutefois, plus encore que les autres stockages, d'être proches des centres d'utilisation. Différents systèmes de stockage sont actuellement développés dans ce but :

- injection d'eau en formation aquifère, les calories étant véhiculées par convection forcée dans une couche aquifère. L'exploitation peut se faire par puits unique, par doublet à puits chaud et froid, dans des aquifères libres ou confinés.

- échangeurs enterrés, les calories étant transférées au sein du stockage par conduction thermique à partir d'un échangeur (tubes horizontaux, radiateurs enterrés ou forages verticaux).

- stockages d'eau chaude en cavités naturelles ou artificielles, revêtues ou non. L'utilisation de jupes

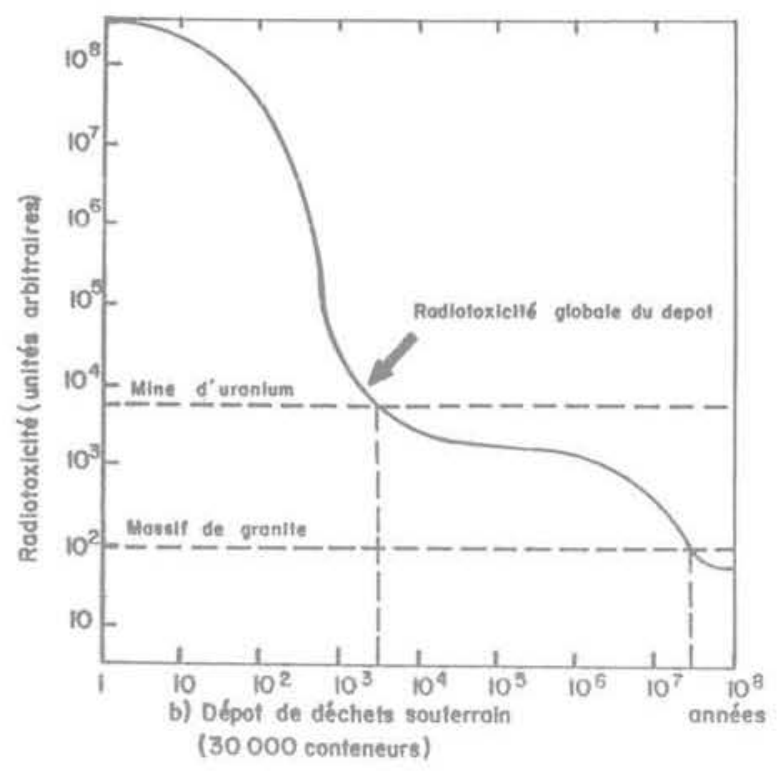

plastiques assurant l'étanchéité des cavités de stockage permet d'envisager l'utilisation de carrières, puits et mines abandonnées près de centres urbanisés [Montjoie, 1981].

Ce type de stockage présente peu de risques d'impact sur l'environnement géologique ou écologique.

Au total, les problèmes posés par des stockages souterrains de produits consommables, dont la durée de vie sera de quelques générations humaines, donnent lieu à des études et des programmes d'auscultation qui permettent de maitriser avec une fiabilité satisfaisante leur impact sur l'environnement. II conviendra tout au plus de contrôler précisèment leur fin d'exploitation et leur abandon, de manière à ce que ces phases ultimes ne se traduisent pas par des désordres soigneusement évités jusqu'alors.

La situation est toute autre en matière d'évacuation définitive de déchets de grande nocivité dans le sous-sol.

\section{L'enfouissement profond des déchets toxiques}

Chaque jour, on produit en France un million de tonnes de déchets de toutes natures. Annuellement, 16 millions de tonnes de déchets industriels renferment des éléments nocifs, dont la moitié est rejetée directement à l'égoût. Deux millions de tonnes de bains et autres concentrés chimiques à forte teneur en substances toxiques et dangereuses sont produits annuellement.

A l'examen de ces chiffres, le million de mètres cubes de déchets radioactifs que les centrales électronucléaires auront produit en l'an 2000 dans notre pays paraît bien raisonnable. En particulier, lorsque l'on sait que plus de 900000 mètres cubes seront des déchets de faible et moyenne activité dont la radiotoxicité ne dépassera pas quelques siècles. Seuls les déchets contaminés par des émetteurs alpha (moins de 40000 mètres cubes en l'an 2000 d'après l'Andra") et les déchets de retraitement de haute activité, qui sont actuellement conservés en piscine pour refroidissement, contiennent la majeure partie de la radioactivité engendrée par l'électronucléaire. Ces derniers ont une durée de vie et une radiotoxicité quasi-illimitées (fig. 4). Ils sont incorporés dans des verres borosilica-

\footnotetext{
- Andra : Agence Nationale pour la Gestion des Déchets Radioactifs.
} 

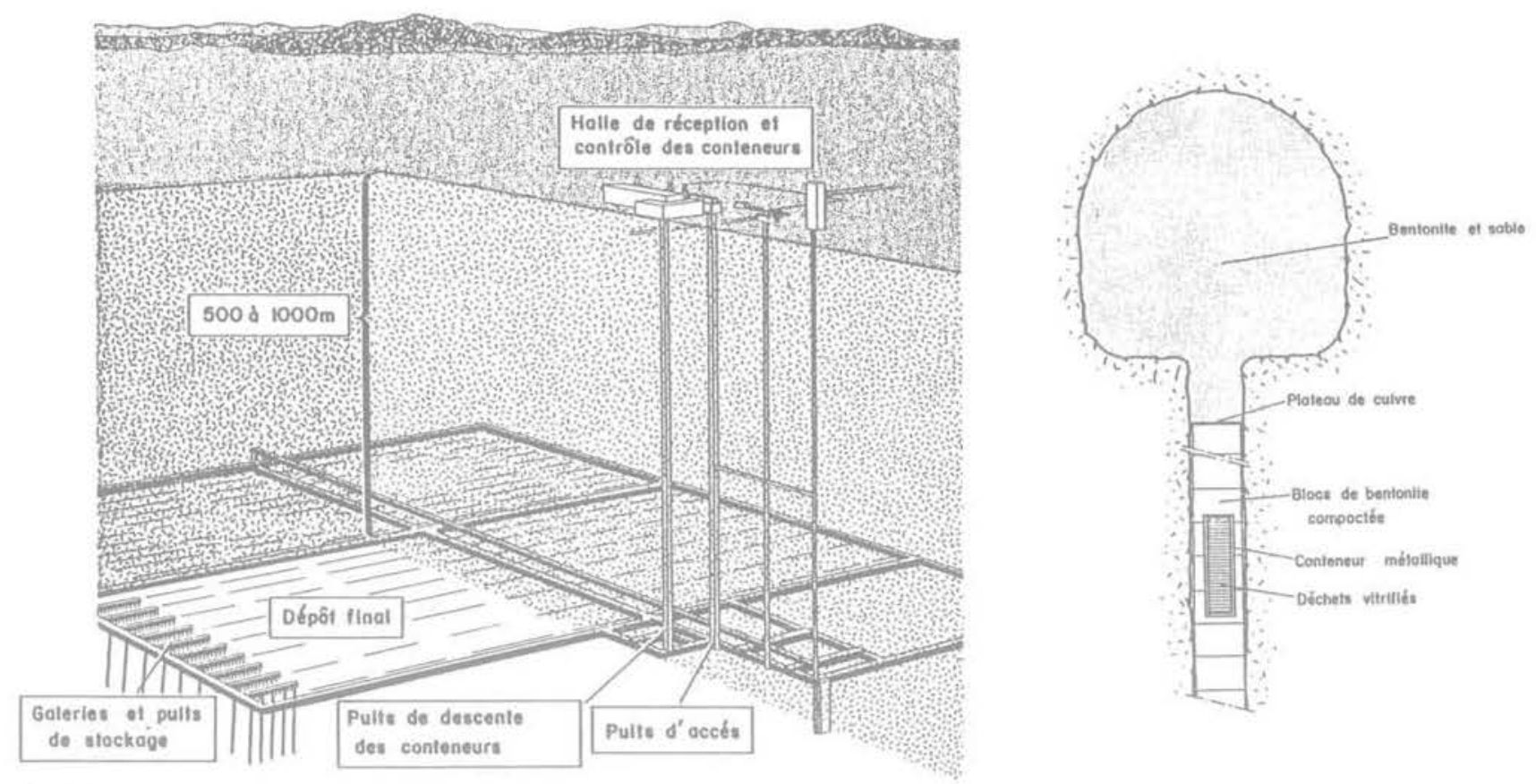

Fig. 5

a) Coupe et perspective de l'installation d'évacuation

b) Coupe de galerie et puits de stockage après fermeture

tés dont le volume total ne dépassera pas quelques milliers de mètres cubes à la fin du siècle. En dépit du laxisme persistant en matière de réglementation sur la gestion des déchets chimiques, les organismes internationaux et nationaux chargés de la radioprotection, coutumiers et respectueux de normes de sûreté rigoureuses, ont jugé que la dispersion des déchets à vie longue dans le sol et les eaux n'est pas biologiquement acceptable. Leur transformation ou leur isolement définitif de la biosphère s'impose.

Des concepts d'élimination variés ont èté envisagés pour isoler ces déchets de la biosphère : évacuation dans l'espace extra-terrestre, destruction des radionucléides à vie longue par transmutation nucléaire, évacuation sur le fond des océans, évacuation sur le soubassement rocheux des calottes glaciaires, injection dans les zones de subduction des plaques océaniques, enfouissement profond en formations géologiques stables. Cette dernière alternative s'est vite imposée comme la plus accessible techniquement et la plus fiable scientifiquement, tout en paraissant économiquement raisonnable.

Le concept d'évacuation en formations géologiques profondes peut répondre *a priori” à un certain nombre d'exigences parmi lesquelles : inaccessibilité, stabilité à long terme, piégeage ou retardement du retour partiel des radionucléides jusqu'à la biosphère.

Les nappes d'eau souterraine ou l'eau interstitielle que l'on rencontre dans toutes les roches de la partie superficielle de la croûte terrestre constitueront le principal facteur potentiel de libération et de transfert des radionucléides jusqu'à la biosphère. C'est pourquoi les formations géologiques retenues jusqu'ici comme milieux de confinement potentiels (sel, argiles et schistes, granites et gneiss, basaltes) ont été choisies pour leurs structures simples, leur abondance, leur faible perméabilité ou porosité en masse, leur conductivité thermique et/ou leur capacité de sorption élevée.

Les projets de dépôts miniers en formations géologiques continentales actuellement élaborés (fig. 5) respectent trois exigences pratiques:
1) Les niveaux d'évacuation des déchets conditionnés à vie longue doivent être les plus profonds possible de manière à augmenter l'isolation des déchets. Toutefois, cette tendance est limitée par le coût de la construction, la stabilité des excavations et la température de travail en galerie qui devient rapidement excessive compte tenu du gradient géothermique naturel. Ces considérations antagonistes ont conduit jusqu'ici à la conception de projets d'évacuation à des profondeurs maximales de l'ordre de 1000 à 1300 mètres, profondeurs qui sont encore très superficielles lorsqu'on les compare à l'épaisseur des plaques continentales ou même à la frange d'exploitation actuelle du sous-sol.

2) Les déchets de retraitement contiennent des produits de fission dont la décroissance se traduit par un dégagement d'énergie thermique considérable au cours des premiers siècles (fig. 6). Pour cela, ils doivent être répartis homogènement dans la formation géologique avec une densité assez faible pour que les contraintes thermiques induites à la paroi et au sein de la masse rocheuse ne mettent en cause la stabilité mécanique et géochimique du massif. Ceci conduit à envisager des volumes de dépôts importants et en particulier une grande extension horizontale (plusieurs kilomètres carrés).

3) Les cavités créées pour la mise en place des conteneurs de déchets à vie longue seront remplies par une série de matériaux, disposés de manière homogène ou en couches successives, en vue d'assurer I'isolement des déchets, la stabilité, "l'étanchéité " et l'inviolabilité du dépôt.

Ces matériaux doivent être sélectionnés seion un critère de stabilité à long terme, face aux phénomènes d'irradiation, d'élévation de température (dans le cas de déchets de haute activité) et d'érosion ou d'altération par l'eau des nappes aquifères (tout particulièrement dans la partie sommitale des puits).

En aucun cas les puits et galeries d'accès ne devront limiter la potentialité de confinement du milieu géologique sélectionné. Si cette question paraît secondaire pour les puits foncés dans des formations 


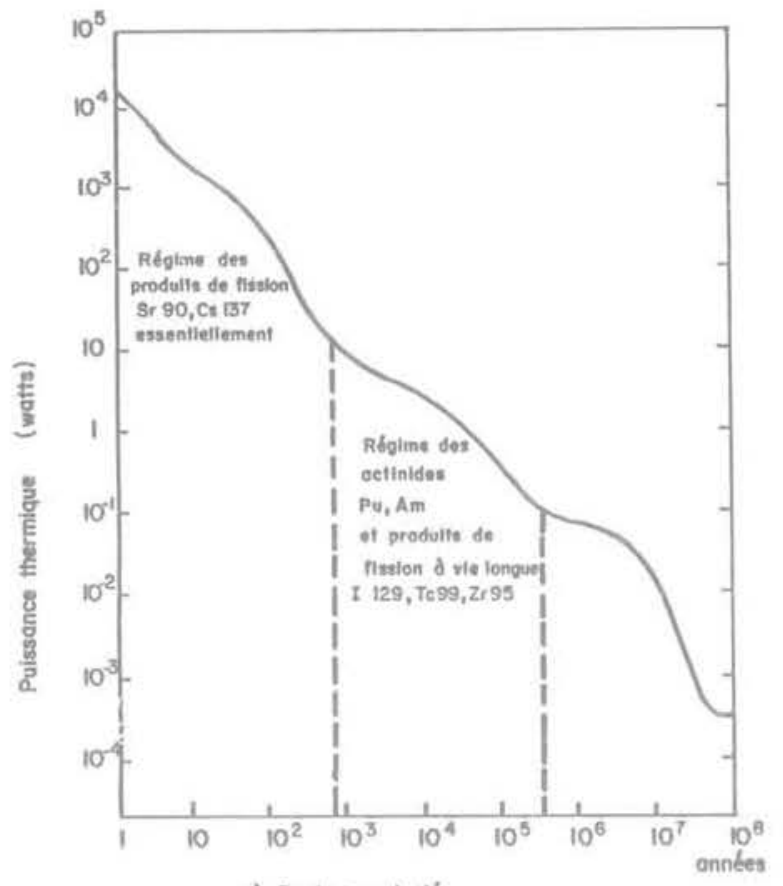

a) Contenaur isol6

argileuses plastiques, elle constitue un problème technologique qu'il serait imprudent de mésestimer pour les autres formations géologiques qui devront être remplies et obturées par des matériaux satisfaisants disponibles en quantités suffisantes et à un coût raisonnable.

A titre d'exemple [Maury, 1981], les déchets de retraitement provenant du fonctionnement du parc électronucléairé français jusqu'à la fin du siècle pourraient être enfouis dans un seul site d'évacuation en milieu granitique. Situé à 1000 mètres de profondeur, le dépôt souterrain aurait 5 kilomètres carrés d'extension horizontale environ, les conteneurs de déchets étant disposés dans des forages de 100 mètres de longueur. Cette hypothèse d'école conduit à envisager l'exécution de 180 kilomètres de galeries et 600 kilomètres de forages. Une telle installation d'évacuation fonctionnerait pendant 80 ans avant que la fermeture définitive du dépôt puisse être envisagée.

Les méthodes d'analyses, de mesures et de simulation mises en œuvre au cours des dernières décennies pour préciser la connaissance des équilibres internes des massifs rocheux, ainsi que l'expérience accumulée, sont satisfaisantes pour l'élaboration de tels projets de construction. En ce sens, la conception d'installations d'enfouissement de déchets nocifs dans le sous-sol ne pose pas de problèmes de construction exceptionnels pour le géotechnicien. II n'en va pas de même pour l'évaluation scientifique de la sureté à long terme du confinement des radionucléides apporté par les barrières technologiques et géologiques retenues dans les projets [Masure, 1981]. Pour la première fois sans doute, la communauté scientifique (géologues, hydrogéologues, géotechniciens, géochimistes, géothermiciens, radiochimistes, métallurgistes, biologistes, ingénieurs civils, etc.) ressent les incertitudes bien connues des géologues, confrontés à des échelles de temps et d'espace sans commune mesure avec celles de l'expérimentation, à des lois de comportement mal appréhendées ou à des milieux mal définis.

Les incertitudes qui sont liées à l'échelle de temps "géologique " imposée par le problème, conduisent à tenir compte de situations, dimensions ou facteurs cinétiques jugés négligeables dans les raisonnements courants: amplitude de l'érosion superficielle à

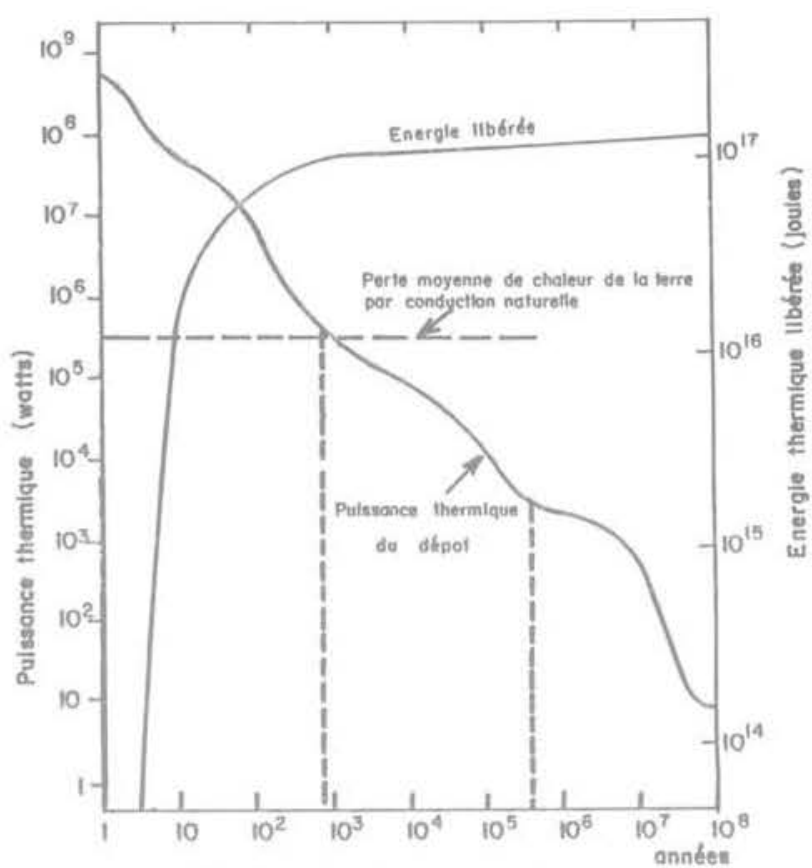

b) D£pot de déchels souterrain

( 30000 conteneurs)

T'échelle du massif par exemple, influence des cycles climatiques lents sur les équilibres internes du milieu, effet à long terme des variations de température et des contraintes sur les conditions d'écoulement des fluides en milieux de très faible perméabilité, évaluation des risques d'intrusion accidentelle dans le dépôt souterrain par les générations futures, etc.

Les incertitudes qui sont liées au dégagement thermique des déchets de retraitement introduisent une dimension nouvelle en matière d'impact des grands ouvrages sur la géosphère, que ce soit des points de vue structural, minéralogique, géochimique, hydraulique ou mécanique. Après la découverte du choc hydraulique induit par les retenues de certains grands barrages, ou par l'injection massive de liquides dans certaines couches profondes, ne doit-on pas craindre un choc thermique ignoré jusqu'ici?

Face à ces incertitudes, il convient aujourd'hui de se référer aux enseignements apportés par l'observation de notre environnement. Pour de nombreux aménagements de surface, le meilleur emplacement est plus ou moins imposé par la nature: défilés pour les barrages et les ponts, vallées et cols pour les voies de communication, chutes d'eau pour l'énergie hydroélectrique. Tous ces sites naturels sont des emplacements privilégiés par des particularités morphologiques, tout comme les gisements métallifères sont privilégiés par leur contexte géologique et géochimique. La connaissance des potentialités de concentration et de piégeage naturel présentées par la géosphère (de nature structurale, stratigraphique, biochimique, capillaire, mécanique ou osmotique) a été à l'origine de l'option que représente l'évacuation des radionucléides à vie longue dans le sous-sol. Les références fournies par l'analyse détaillée des conditions naturelles de piégeage des éléments constitutifs de l'écorce terrestre doivent être exploitées, aussi bien dans la conception des projets d'évacuation et de confinement des déchets que dans la définition de critères de sélection des sites potentiellement favorables. Dans ce sens, les responsables du stockage souterrain d'hydrocarbures se sont déjà inspirés des conditions de piégeage naturel (fig. 2) pour rechercher des structures convenables. Cette notion gagnerait à être utilisée dans la recherche de sites d'évacuation 
notamment pour les déchets de faible et moyenne activité comme pour les déchets chimiques très toxiques: structures anticlinales ou litées étanches, zones à gradient hydraulique nul, barrières capillaires, filtres géochimiques, etc.

Le concept de barrières multiples s'opposant à la migration des radionucléides dans la géosphère a donné lieu à de nombreuses recherches sur les barrières artificielles qui pourraient être disposées autour des conteneurs dans les puits forés (barrières géochimiques ou métalliques). Ce concept doit être enrichi par la recherche de solutions de traitement $d u$ milieu géologique entourant le dépôt en vue de limiter (ou éliminer) l'accès de l'eau souterraine dans le dépôt. L'aptitude de certaines formations à voir leurs caractéristiques naturelles améliorées par des procédés simples et dont la pérennité est assurée devrait constituer un autre critère de sélection préférentielle. A titre d'exemple, le traitement de certains milieux rocheux peut être très efficace par injection de silice ou de précipités dans les fissures ou les pores, par drainage des abords du dépôt ou par utilisation de barrières capillaires.

La définition de projets fiables de confinement à long terme des déchets de retraitement ne sera possible que lorsque sera mieux connu l'impact thermique de dépôts de grande capacité. Si l'élévation de température dans le massif entourant le dépôt peut être diminuée par une limitation de la densité des conteneurs mis en place, l'énergie thermique dégagée en quelques décennies atteindra, dans le cadre de certains projets actuels de dépôts, une dimension comparable à celle de phénomènes géologiques non négligeables. L'expérience apportée par l'exploitation croissante des ressources géothermiques pourra sans doute améliorer les connaissances des spécialistes en ce domaine dans les années à venir. En attendant, il n'est pas possible d'affirmer que la probabilité est nulle de voir l'impact des dépôts de déchets de retraitement se traduire, dans certains cas particuliers, par des secousses sismiques dans les massifs rigides, par une forte migration de l'eau interstitielle dans les couches argileuses plastiques* pouvant conduire à u. - e destruction de la structure interne de la formation, ou par des phènomènes de soulèvement néodiapiriques dans les dômes salins qui pourraient rompre l'étanchéitè des couches imperméables qui ont empêché jusqu'ici la dissolution des masses salées par l'eau des nappes aquifères superficielles.

- Quif ont une teneur en eau considérable: 20 à $35 \%$.
Face à ces incertitudes inhérentes aux opérations nouvelles en matière de génie géologique, il conviendrait de réaliser assez rapidement quelques installations pilotes expérimentales et réversibles simulant l'ouvrage final, en vue d'analyser l'impact réel du dépôt sur la géosphere, avec auscultation détaillée du massif et de l'ouvrage pendant plusieurs décennies. Ce n'est qu'après une telle phase expérimentale que la définition de critères de sélection de sites d'évacuation de déchets de retraitement pourra être assurée avec pertinence.

Ces quelques réflexions suffisent à démontrer que les problèmes scientifiques et techniques posés par l'enfouissement des déchets très nocifs dans le sous-sol, en vue de leur élimination définitive de la biosphère, présentent bien des aspects complémentaires de ceux que les spécialistes de stockage souterrain de substances récupérables ont à résoudre depuis 25 ans. Aussi, d'importants programmes de recherches internationales sont-ils coordonnés de manière efficace par des organismes comme la Commission des Communautés Européennes (Bruxelles), I'Agence de I'Énergie Atomique de I'O.C.D.E. (Paris) et l'Agence Internationale pour l'Énergie Atomique (Vienne). On peut espérer que les progrès que permettront ces programmes en matière de connaissance de l'environnement géologique et de protection de l'environnement écologique favoriseront la mise en cuvre d'une politique de gestion plus rigoureuse des quantités inquiétantes de déchets chimiques toxiques qui sont actuellement produits dans les pays industrialisés. II est probable que le recours à des solutions d'enfouissement profond dans le sous-sol connaîtra alors un développement considérable auquel les géologues et les géotechniciens doivent se préparer dès aujourd'hui.

\section{Exploitation, occupation et gestion du sous-sol}

Dans un pays de faible superficie comme la France, le recours au sous-sol ne constitue plus une simple alternative, comme il l'a longtemps été en matière d'aménagement. II devient bien au contraire une nécessité impérieuse en matière de stockage de grandes quantités de produits consommables et de calories, tout comme it semble devoir le devenir en matière de confinement de déchets très toxiques.

Depuis les premières traces d'exploitation du sous-sol à Maastricht; il y a plus de 5000 ans, l'exploitation minière s'est approfondie, avec des records à plus de 3000 mètres (Afrique du Sud). Les réservoirs d'hydro-
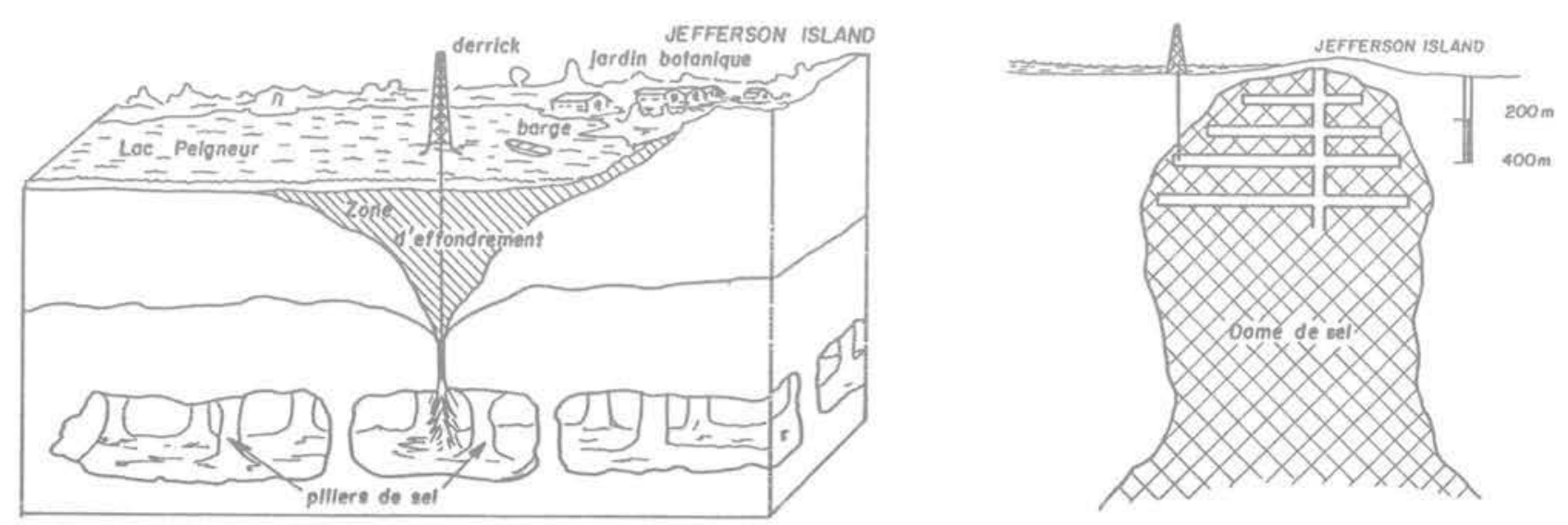

Fig. 7 
carbures sont recherchés jusqu'à 7000 mètres de profondeur. Si l'exploitation des eaux souterraines conduit rarement à effectuer des forages de plus de 1500 mètres, l'exploitation des ressources géothermiques, qui connaît une forte croissance, atteint fréquemment des profondeurs de 5000 mëtres. Le sondage de reconnaissances géologiques le plus long réalisé à ce jour est de 9760 mètres (presqu'île de Kola, U.R.S.S., 1978), tandis que la mine à ciel ouvert (open-pit) la plus profonde a atteint -900 mètres (Canada).

Les projets de stockage souterrain d'hydrocarbures (fig. 5) se situent généralement entre quelques dizaines (liquides) ou centaines de mètres (gaz) et 2000 mètres (cavité de dissolution saline, Eminence, U.S.A.). II est donc évident que les concepts actuels d'évacuation de déchets radioactifs se situent dans une tranche très superficielle de l'épiderme de la Terre*, quelque peu altérée par les activités humaines.

La profondeur des cavités minées est limitée par les problèmes de stabilité des excavations, par la chaleur naturelle dégagée en souterrain et par des considérations économiques. Des risques d'encombrement des sites de stockage souterrain en grandes cavités ne sont donc pas négligeables [J. Bregeon et P. Duffaut, 1981] dans un proche avenir et les massifs qui ne renferment pas de ressources minérales, organiques ou hydriques ne sont pas à l'abri d'une utilisation rapide de leurs ressources spatiales ou géothermiques. Une destruction irréversible des capacités de confinement de certains contextes favorables au stockages ou à l'évacuation est également à craindre.

Les exemples de coexistence difficile entre cavités souterraines sont déjà nombreux. Ainsi, la construction de tunnels hydro-électriques dans les Alpes françaises s'est heurtée à des difficultés liées à la proximité de nombreuses petites mines de charbon abandonnées depuis longtemps. Les effets catastrophiques de lintrusion accidentelle d'un forage dans une mine ont récemment été illustrés de manière spectaculaire aux États-Unis, en Louisiane (fig. 7). Le 20 novembre 1980, à Jefferson Island, les eaux du lac Peigneur disparaissaient dans un forage pétrolier en cours de perforation, détruisant par leur tourbillon plate-forme de forage, derricks, barges; remorqueurs et maisons. Ce forage explorait les abords d'un dôme de sel, alors qu'une mine exploitait le même diapir. La pénétration du forage dans la mine à $400 \mathrm{~m}$. de profondeur allait favoriser l'intrusion d'eau, la dissolution des piliers et l'effondrement partiel de la mine. Bien que les dégâts fussent considérables, il n'y eut pas de victime.

Toutes ces remarques démontrent l'importance qu'il y a d'organiser une gestion rationnelle des ressources du sous-sol. Celui-ci n'apporte pas seulement des ressources minérales, organiques et géothermiques (l'eau, les matériaux, les minerais, les hydrocarbures, le charbon), il apporte aussi des ressources spatiales et des ressources de confinement. Tout comme pour les ressources minières, incluant les eaux chaudes et l'eau, dont l'inventaire pour la France est réalisé par le Bureau de Recherches Géologiques et Minières, un véritable inventaire des contextes géologiques particulièrement favorables au piégeage et au confinement des décrets nocifs est à présent indispensable, ces déchets devant être considérés comme un minerai artificiel. Un tel inventaire devrait être intégré dans une

- Alors que lépaisseur moyenne de la croôte terrestre est de 40 kilomètres. banque de données centralisée regroupant tous les sites sélectionnés pour le stockage souterrain en grandes cavités convenant spécifiquement à chaque type de produit (hydrocarbures liquides ou gazeux, air comprimé, eau chaude, déchets chimiques, etc.) afin de définir des priorités et établir des réservations, des concessions. La gestion d'une banque de données sur les ouvrages et sites souterrains éliminerait les risques d'intrusion accidentelle et les difficultés de coexistence.

La prise en compte globale des intérêts de l'exploitation du sous-sol (ressources minérales, organiques et géothermiques) et de l'occupation du sous-sol (stockages, confinement, abris) permettrait d'en optimiser la gestion et d'éviter des conflits d'intérêt préjudiciables, par des mesures réglementaires, des incitations, des interdictions. Un véritable bilan prospectif en matière d'occupation de l'espace souterrain comparant les besoins et les ressources pourrait conduire à terme à une entente entre partenaires nationaux qui éviterait de passer sans transition de l'abondance à la pénurie, situation que nous connaissons déjà, au niveau international, en matière d'exploitation des ressources du sous-sol.

Cet objectif ne peut être atteint sans un ensemble de démarches convergentes de l'État et des différents maîtres d'ouvrages, de l'État et du public qui doit être informé et sensibilisé à cette nécessité, qui doit en comprendre les inconvénients, mais aussi les avantages et les impératifs.

\section{Références bibliographiques}

J. Bregeon, P. Duffaut (1981) La variante souterraine comme solution aux problèmes d'environnement, Deuxièmes Journées nationales géotechniques « Environnement et Géotechnique ", Nantes, 11-13 mars 1981.

P. Duffaut (1981) Prospection des usages de l'espace souterrain en Europe, Symposium sur l'Usage industriel du sous-sol, Société espagnole de mécanique des sols, Madrid, 7-9 avril 1981.

J. Lacoste, P. Bérest (1981) Politique du ministère de I'Industrie en matière de sécurité et protection de l'environnement dans les stockages souterrains d'hydrocarbures, Deuxièmes Journées nationales géotechniques "Environnement et Géotechnique ", Nantes, 11-13 mars 1981.

Ph. Masure (1981) L'environnement et les concepts d'évacuation de déchets radioactifs à vie longue en formations géologiques continentales, Deuxièmes Journées nationales géotechniques "Environnemet et Géotechnique ", Nantes, 11-13 mars 1981.

V. Maury (1981) Le stockage souterrain : conception et réalisation. Applications aux énergies conventionnelles, nouvelles, aux déchets et à divers produits, Conférence à l'Institut du bâtiment et des travaux publics, Paris, 31 mars 1981, à paraître dans les Annales de I'I.T.B.T.P.

A. Monjoie (1981) Stockage de chaleur dans les excavations à ciel ouvert ou souterraines, Deuxiemes Journées nationales géotechniques «Environnement et géotechnique $»$ Nantes, 11-13 mars 1981. 
